\title{
ESTRUTURA HORIZONTAL E VERTICAL DO COMPONENTE ARBÓREO EM FASE DE REGENERAÇÃO NATURAL NA MATA SANTA LUZIA, NO MUNICÍPIO DE CATENDE-PE ${ }^{1}$
}

\author{
Wegliane Campelo da Silva², Luiz Carlos Marangon ${ }^{3}$, Rinaldo Luiz Caraciolo Ferreira ${ }^{3}$, Ana Lícia Patriota Feliciano ${ }^{3}$, \\ Perseu da Silva Aparício² e Roberto Felix Costa Junior ${ }^{2}$
}

\begin{abstract}
RESUMO - O estudo foi desenvolvido no fragmento denominado Mata Santa Luzia, situado no Município de Catende, PE, nas coordenadas $8^{\circ} 72^{\prime} 49^{\prime \prime}$ S e 3572'47" W, altitude de $239 \mathrm{~m}$, com os objetivos de avaliar a regeneração natural total de espécies arbóreas no fragmento florestal e verificar a diversidade e estrutura das espécies nessa área. Para a estimativa da regeneração natural das espécies arbóreas no fragmento, foram locadas de forma sistemática 15 subparcelas permanentes de $25 \mathrm{~m}^{2}$ ( 5 x $5 \mathrm{~m}$ ), equidistantes em $50 \mathrm{~m}$. O nível de inclusão adotado foi de CAP $<15 \mathrm{~cm}$ e as medições de altura (h), divididas em três classes, em que a classe 1 contemplou indivíduos com $1,0<\mathrm{h}<2,0 \mathrm{~m}$, a classe 2 com indivíduos 2,0 $<\mathrm{h}<3,0 \mathrm{~m}$ e a classe 3 com indivíduos com $\mathrm{h}>3,0 \mathrm{~m}$. No fragmento foram amostrados 107 indivíduos vivos, pertencentes a 25 famílias botânicas e 36 táxons. Dessas, 33 foram identificadas em nível de espécie, duas em nível de gênero e uma se apresentou como indeterminada. As cinco espécies com maiores valores para regeneração natural total (RNT) foram assim distribuídas: Rheedia gardneriana Planch. \& Triana (9,9\%), Cupania racemosa (Vell.) Radlk (7,7\%), Brosimum discolor Schott (7,4\%), Vismia guianensis (Aubl.) Pers. (6,1\%) e Protium heptaphyllum (Aubl.) Marchand (5,9\%). Provavelmente, essas espécies no futuro serão as principais responsáveis pela manutenção da estrutura e fisionomia da floresta.
\end{abstract}

Palavras-chave: Fitossociologia, Floresta Ombrófila Densa e Mata Atlântica.

\section{ESTRUCTURE HORIZONTAL E VERTICAL IN THE ARBOREO COMPONENT IN NATURAL REGENERATION FASE IN A MATA SANTA LUZIA, CATENDE'S CITY-PE}

\begin{abstract}
The study was developed in the fragment situated in the Catende's-PE city (Mata Santa Luzia - 8'72'49" S e 35072'47" Wat $142 \mathrm{~km}$ of the capital, with $239 \mathrm{~m}$ altitude. The present work aims to study and evaluated the total natural regeneration of arboreal species in the forest fragment and to verify the diversity and structure of the species in the same one. For estimate the natural regeneration, were made systematically 20 subunits (samples with $5 \times 5 \mathrm{~m}$ ), with 50 meters between the ones. The regeneration study $(C A P<15 \mathrm{~cm})$ was developed with heights divided in three categories (1 - individuals with minimum heights $1<2 \mathrm{~m} ; 2$ - individuals with minimum heights $2<3 \mathrm{~m}$; 3 - individuals with heights $>3 \mathrm{~m}$ ). - individuals with heights $>3 \mathrm{~m}$ ). In fragment was observed 25 botanical families and 36 arboreal táxons inside 107 individuals. 33 had been identified in level of species, 2 in sort level and 1 are not identify. five species obtained bigest values for total natural regeneration of the samples population (RNT) was descript following way: Rheedia gardneriana Planch. \& Triana (9,9\%), Cupania racemosa (Vell.) Radlk (7,7\%), Brosimum discolor Schott (7,4\%), Vismia guianensis (Aubl.) Pers. (6,1\%), Protium heptaphyllum (Aubl.) Marchand (5,9\%). These species probably they will be main responsible for the maintenance of forest's structure and physiognomy.
\end{abstract}

Keywords: Phytossociology, Humid Forest and Atlantic Forest.

\footnotetext{
${ }^{1}$ Recebido em 29.03.2008 e aceito para publicação em 02.03.2010.

${ }^{2}$ Universidade do Estado do Amapá, UEAP, Brasil. E-mail: <wegliane@hotmail.com>.

${ }^{3}$ Universidade Federal Rural de Pernambuco, UFRPE, Brasil.
} 


\section{INTRODUÇÃO}

Tradicionalmente, os trabalhos em florestas nativas têm sido motivados pela falta de informações de inúmeras espécies, principalmente as de porte arbóreo. No entanto, embora de importância crescente, há limitação de informações sobre as comunidades que compõem a floresta e das espécies arbóreas existentes, além de não se conhecer também os fenômenos inter e intraespecíficos que ocorrem na floresta ou, mesmo, em espécies isoladas. Além disso, a má utilização dos recursos florestais têm proporcionado sérios danos a esses recursos, comprometendo seriamente a biodiversidade (MARANGON et al., 2003).

No Brasil, a formação das florestas secundárias é atribuída à expansão da fronteira agrícola, aos projetos de urbanização e industrialização e à mineração. Também é formada pela exploração seletiva de madeiras e pelo corte raso para a realização da agricultura itinerante, o que causa a abertura de grandes clareiras e o surgimento da vegetação secundária (RONDON NETO et al., 2000).

Nas áreas onde ocorre a sucessão secundária, a composição florística vai se modificando, tornando a comunidade cada vez mais complexa e diversificada. Portanto, os estudos da regeneração natural são necessários para que os mecanismos de transformação da composição florística e estrutura possam ser compreendidas. Tais conhecimentos constituem uma ferramenta básica para a tomada de medidas que visem à aceleração e direcionamento do processo de sucessão secundária, seja para preservação ou produção comercial.

Atualmente, as florestas em estado de perturbação são alvo preferencial de estudos ou de perfilhação de medidas conservacionistas, abrangendo grande parte dos remanescentes no mundo, de maneira a proporcionar maior entendimento da dinâmica e das características fisionômicas da vegetação em fase de regeneração natural.

Juntamente com estudos fitossociológicos, as análises da regeneração natural de espécies arbóreas têm sido fundamental para a adoção de planos de restauração, conservação e manejo de inúmeros fragmentos florestais, mantendo as características da comunidade e composição de espécies e, ainda, quando associados a estudos e avaliações periódicas, é possível estimar o nível de estabilidade ecológica e a velocidade de recuperação da floresta, sendo esses diretamente influenciados pela intensidade de perturbação.
Durante o estudo da regeneração natural podem ser obtidas informações sobre autoecologia, estádio sucessional, efeitos da exploração ou colheita florestal, entre outras informações importantes que norteiam as intervenções silviculturais previstas nos planos de manejo (HIGUCHI et al., 1985). Desse modo, a regeneração natural permite análise efetiva para diagnosticar o estado de conservação do fragmento e a resposta ao manejo, uma vez que representa o conjunto de indivíduos capazes de serem recrutados para os estádios posteriores. Portanto, o estudo da regeneração das florestas constitui-se num tema de relevância para a preservação, conservação e recuperação das florestas.

O fragmento Mata Santa Luzia foi escolhido para compor este trabalho, por estar inserido no Município de Catende, por ser uma das poucas regiões estudados da floresta ombrófila densa, que se encontram situados na Zona da Mata Sul pernambucana, e por obter uma das maiores áreas entre os remanescentes locais e possuir melhor acessibilidade. Este trabalho visou estudar e verificar o comportamento das espécies florestais arbóreas que se encontram em fase de regeneração no fragmento Mata Santa Luzia, com o objetivo de subsidiar informações de conhecimento florístico, fitossociológico e do estado de regeneração total da área.

\section{MATERIAL E MÉTODOS}

\subsection{Caracterização da área}

O estudo foi desenvolvido no fragmento denominado Mata Santa Luzia (27,47 ha), localizado no engenho Santa Luzia do complexo da Usina Catende. O fragmento encontra-se na mesorregião da mata pernambucana, a 239 m de altitude, mais precisamente na microrregião da mata úmida, a $142 \mathrm{~km}$ da capital, nas coordenadas 872'49" S e 3572'47" W. O acesso é feito pela Br101 Sul e pela PE-126. A temperatura anual varia entre 22 e $26^{\circ} \mathrm{C}$, e a precipitação média anual é de $1.414 \mathrm{~mm}$. O relevo varia de ondulado a fortemente ondulado. O município está inserido na bacia hidrográfica do rio Uma. O clima é do tipo As’, tropical chuvoso com verão seco e estação chuvosa adiantada para o outono, antes do inverno, segundo a classificação de Köeppen (CONDEPE, 1987).

A formação vegetacional da área estudada no Município de Catende, Pernambuco, corresponde à Floresta Ombrófila Densa, segundo Silva et al. (2007). 
Os solos predominantes na área geográfica do município são classificados como Latossolo Vermelho distrófico e o Nitossolo Vermelho associado ao latossolo (EMBRAPA, 2005). São em geral solos de baixa fertilidade, de textura argilosa, podendo conter rochas bem granitizadas.

\subsection{Análise da Estrutura da Regeneração Natural}

Para a estimativa da regeneração natural das espécies arbóreas, foram locadas de forma sistemática 15 subunidades de $25 \mathrm{~m}^{2}$ ( $\left.5 \times 5 \mathrm{~m}\right)$, com base na metodologia empregada por Volpato (1994). Essas subunidades foram implementadas no centro de 15 unidades amostrais de $250 \mathrm{~m}^{2}$ (10 x $\left.25 \mathrm{~m}\right)$, anteriormente locadas de forma permanente para o estudo da fitossociologia da comunidade arbórea adulta, equidistantes $50 \mathrm{~m}$.

Os indivíduos mensurados receberam placas de alumínio com numeração crescente. Para mensurar a altura dos indivíduos que apresentaram até $2,0 \mathrm{~m}$, foi utilizada trena de bolso. Quando necessário, estimaram-se as demais com varas moduladas de 2,0 m.

As análises foram estabelecidas com base no nível de inclusão, proposto por Finol (1971 adaptada por MARANGON, 1999), em que foram mensurados indivíduos que apresentaram CAP $\leq 15 \mathrm{~cm}$ e alturas correspondentes divididas em classes, em que a classe 1 contemplou indivíduos com $1,0 \leq \mathrm{h} \leq 2,0 \mathrm{~m}$, a classe 2 com indivíduos $2,0<\mathrm{h} \leq 3,0 \mathrm{~m}$ e a classe 3 com indivíduos com h > 3,0 m.

Optou-se pela altura mínima de 1,0 m para análise da regeneração de espécies arbóreas, pois nessa altura as espécies apresentam melhor definição da sua caracterização morfológica, permitindo identificação mais confiável.

O reconhecimento das espécies foi realizada no campo, e todo o material botânico foi coletado para identificação posterior no Herbário Dardáno de Andrade Lima - IPA (Empresa Pernambucana de Pesquisa Agropecuária), seguindo o sistema de classificação de Cronquist (1988), conforme o empregado por Marangon et al. (2003).

Para cada espécie foram estimados os parâmetros absolutos e relativos de frequência e densidade, em cada classe de altura pré-estabelecida utilizando o Microsoft EXCEL 2003 e o Software MATA NATIVA, Versão 2.0. Com base nesses parâmetros, estimou-se a regeneração natural por classe de altura para RNC1 (Regeneração Natural na Classe de Altura 1); RNC2 (Regeneração Natural na Classe de Altura 2); RNC3 (Regeneração Natural na Classe de Altura 3); em que para isso foi usada a seguinte expressão (VOLPATO, 1994):

em que:

$$
R N C_{i j}=\frac{D R_{i j}+F r_{i j}}{2}
$$

RNCij = estimativa da regeneração natural da iésima espécie na j-ésima classe de altura de planta, em percentagem;

DRij = densidade relativa em percentagem para a i-ésima espécie na j-ésima classe de altura de regeneração natural; e

FRij = frequência relativa em percentagem para i-ésima espécie na j-ésima classe de regeneração natural.

Calculado o índice de regeneração por classe de altura para cada espécie, foi realizado o cálculo da estimativa da RNT (Regeneração Natural Total da População Amostrada), utilizando-se da soma dos índices de regeneração natural por classe de altura, dividida pelo número de classes, conforme Finol (1971):

$$
R N T_{i}=\sum\left(R N C_{i j}\right) / 3
$$

em que:

RNTi = estimativa da regeneração natural total da i-ésima espécie; e

RNCij = estimativa da regeneração natural da iésima espécie na j-ésima classe de altura de planta.

Para análise da suficiência amostral, utilizou-se o procedimento REGRELRP (Regressão Linear com Resposta em Platô) do Sistema para Análise Estatística e Genética - SAEG v.5.0 (VOLPATO, 1994; GAMA et al., 2002; NAPPO et al., 1999; SILVA et al., 2007). O gráfico foi gerado por meio do "software Microsoft EXCEL for Windows ${ }^{\mathrm{TM}}$ 2000”.

\section{RESULTADOS}

Por meio do estudo da suficiência amostral da análise de regressão com resposta em platô, verificou-se que 15 parcelas $\left(500 \mathrm{~m}^{2}\right)$ foram suficientes para representar a composição florística da regeneração da Mata Santa Luzia (Figura 1).

Revista Árvore, Viçosa-MG, v.34, n.5, p.863-869, 2010 


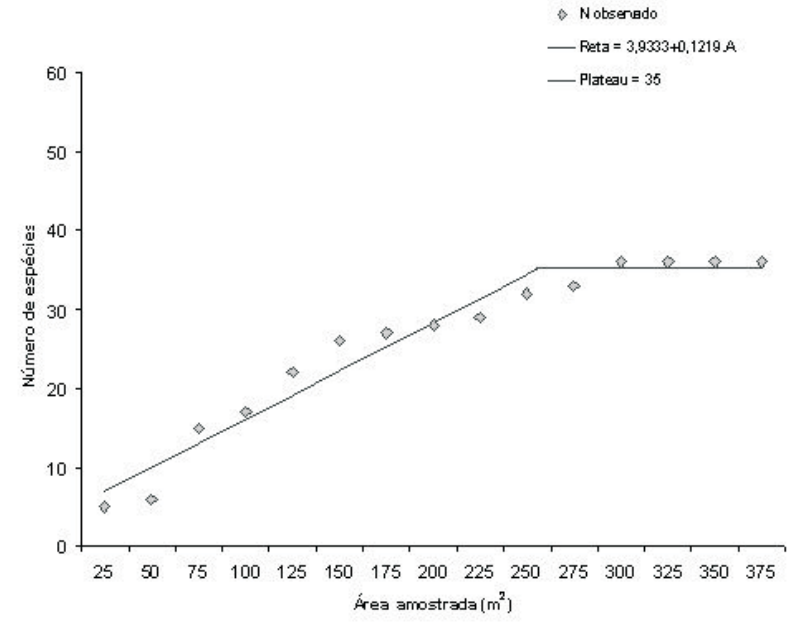

Figura 1 - Determinação da suficiência amostral utilizando o procedimento REGRELRP do SAEG para o estudo da regeneração natural, no fragmento Mata Santa Luzia, Catende, PE.

Figure 1 - Determination of samply sufficient, utilizing the REGRELRP method of SAEG, for natural regeneration study's, Mata Santa Luzia fragment, Catende's city, $P E$.
No fragmento Mata Santa Luzia foram amostrados 107 indivíduos vivos, pertencentes a 25 famílias botânicas, 32 gêneros e 36 táxons (33 táxons foram identificados em nível de espécie, dois em nível de gênero e um indeterminado). As famílias que apresentaram maior destaque em número de espécies foram Clusiaceae (17), Sapindaceae (11), Anacardiaceae (9), Moraceae (8) e Burseraceae (7), e juntas elas representam 36,5\% da regeneração natural.

As espécies que se destacaram com maior número de indivíduos foram, respectivamente: Rheedia gardneriana Planch. \& Triana (10), Cupania racemosa (Vell.) Radlk. (8), Brosimum discolor Schott. (7), Protium heptaphyllum (Aubl.) Marchand (7) e Vismia guianensis (Aubl.) Pers. (7).

As 10 espécies de maiores valores de regeneração natural total da população amostrada (Tabela 1), em ordem decrescente, foram as seguintes: Rheedia gardneriana Planch. \& Triana (9,9\%), Cupania racemosa (Vell.) Radlk (7,7\%), Brosimum discolor Schott (7,4\%), Vismia guianensis (Aubl.) Pers. (6,1\%), Protium heptaphyllum (Aubl.) Marchand (5,9\%), Cordia nodosa

Tabela 1 - Estimativa da Regeneração Natural Total da População Amostrada (RNT) por classe de altura nas subunidades amostrais do fragmento Mata Santa Luzia, em Catende, PE, em que DR = Densidade Relativa; FR = Frequência Relativa; RNC1 = Regeneração Natural na Classe 1 de altura; RNC2 = Regeneração Natural na Classe 2 de altura; e RNC3 = Regeneração Natural na Classe 3 de altura

Table 1 - Estimate of total natural regeneration of the samples population (RNT) height class in samples Mata Santa Luzia fragment, Catende's city, $P E, D R=$ Relative Density; $F R=$ Relative Frequency; $R N C 1=$ Natural Regeneration height class 1; RNC2 = Natural Regeneration height class 2 and RNC3 = Natural Regeneration height class 3.

\begin{tabular}{|c|c|c|c|c|c|c|c|c|c|c|}
\hline Nome Científico & $\begin{array}{c}\text { DR1 } \\
(\%)\end{array}$ & $\begin{array}{l}\text { FR1 } \\
(\%)\end{array}$ & $\begin{array}{c}\text { RNC1 } \\
(\%)\end{array}$ & $\begin{array}{c}\text { DR2 } \\
(\%)\end{array}$ & $\begin{array}{l}\text { FR2 } \\
(\%)\end{array}$ & $\begin{array}{c}\text { RNC2 } \\
(\%)\end{array}$ & $\begin{array}{l}\text { DR3 } \\
(\%)\end{array}$ & $\begin{array}{l}\text { FR3 } \\
(\%) \\
\end{array}$ & $\begin{array}{c}\text { RNC3 } \\
(\%)\end{array}$ & $\begin{array}{c}\text { RNT } \\
(\%)\end{array}$ \\
\hline Rheedia gardneriana Planch. \& Triana & 10,8 & 11,1 & 11,0 & 15,4 & 12,5 & 13,9 & 4,6 & 4,9 & 4,7 & 9,9 \\
\hline Cupania racemosa (Vell.) Radlk. & 5,4 & 5,6 & 5,5 & 7,7 & 8,3 & 8,0 & 9,1 & 9,8 & 9,4 & 7,6 \\
\hline Brosimum discolor Schott & 5,4 & 5,6 & 5,5 & 11,5 & 12,5 & 12,0 & 4,6 & 4,9 & 4,7 & 7,4 \\
\hline Vismia guianensis (Aubl.) Pers. & 10,8 & 8,3 & 9,6 & 3,9 & 4,2 & 4,0 & 4,6 & 4,9 & 4,7 & 6,1 \\
\hline Protium heptaphyllum (Aubl.) Marchand & 8,1 & 8,3 & 8,2 & 0,0 & 0,0 & 0,0 & 9,1 & 9,8 & 9,4 & 5,9 \\
\hline Cordia nodosa Lam. & 2,7 & 2,8 & 2,7 & 11,5 & 12,5 & 12,0 & 2,3 & 2,4 & 2,4 & 5,7 \\
\hline Thyrsodium spruceanum Benth. & 8,1 & 8,3 & 8,2 & 0,0 & 0,0 & 0,0 & 6,8 & 7,3 & 7,1 & 5,1 \\
\hline Quiina sp. & 5,4 & 5,6 & 5,5 & 7,7 & 4,2 & 5,9 & 2,3 & 2,4 & 2,4 & 4,6 \\
\hline Siparuna guianensis Aubl. & 5,4 & 5,6 & 5,5 & 3,9 & 4,2 & 4,0 & 2,3 & 2,4 & 2,4 & 4,0 \\
\hline Tapirira guianensis Aubl. & 0,0 & 0,0 & 0,0 & 7,7 & 8,3 & 8,0 & 2,3 & 2,4 & 2,4 & 3,5 \\
\hline Inga thibaudiana DC. & 5,4 & 5,6 & 5,5 & 3,9 & 4,2 & 4,0 & 0,0 & 0,0 & 0,0 & 3,2 \\
\hline Miconia prasina (Sw.) DC. & 2,7 & 2,8 & 2,7 & 3,9 & 4,2 & 4,0 & 2,3 & 2,4 & 2,4 & 3,0 \\
\hline Myrcia rostrata DC. & 2,7 & 2,8 & 2,7 & 3,9 & 4,2 & 4,0 & 2,3 & 2,4 & 2,4 & 3,0 \\
\hline Amaioua guianensis Aubl. & 5,4 & 5,6 & 5,5 & 0,0 & 0,0 & 0,0 & 2,3 & 2,4 & 2,4 & 2,6 \\
\hline Cupania revoluta Rolfe & 2,7 & 2,8 & 2,7 & 3,9 & 4,2 & 4,0 & 0,0 & 0,0 & 0,0 & 2,3 \\
\hline Dialium guianense (Aubl.) Sandwith & 2,7 & 2,8 & 2,7 & 3,9 & 4,2 & 4,0 & 0,0 & 0,0 & 0,0 & 2,3 \\
\hline Miconia albicans (Sw.) Triana & 2,7 & 2,8 & 2,7 & 3,9 & 4,2 & 4,0 & 0,0 & 0,0 & 0,0 & 2,3 \\
\hline
\end{tabular}


Tabela 1 - Cont.

Table 1 - Cont.

\begin{tabular}{|c|c|c|c|c|c|c|c|c|c|c|}
\hline Guapira opposita (Vell.) Reitz & 2,7 & 2,8 & 2,7 & 0,0 & 0,0 & 0,0 & 4,6 & 2,4 & 3,5 & 2,1 \\
\hline Eriotheca gracilipes (K. Schum.) A. Robyns & 2,7 & 2,8 & 2,7 & 0,0 & 0,0 & 0,0 & 2,3 & 2,4 & 2,4 & 1,7 \\
\hline Ocotea gardneri (Meisn.) Mez & 0,0 & 0,0 & 0,0 & 0,0 & 0,0 & 0,0 & 4,6 & 4,9 & 4,7 & 1,6 \\
\hline Pouteria grandiflora (A. DC.) Baehni & 0,0 & 0,0 & 0,0 & 0,0 & 0,0 & 0,0 & 4,6 & 4,9 & 4,7 & 1,6 \\
\hline Tabebuia impetiginosa (Mart. ex DC.) Standl. & 0,0 & 0,0 & 0,0 & 0,0 & 0,0 & 0,0 & 4,6 & 4,9 & 4,7 & 1,6 \\
\hline Nectandra cuspidata Nees \& Mart. & 0,0 & 0,0 & 0,0 & 0,0 & 0,0 & 0,0 & 6,8 & 2,4 & 4,6 & 1,5 \\
\hline $\begin{array}{l}\text { Inga capitata Desv. } \\
\text { Xylopia frutescens Aubl. }\end{array}$ & $\begin{array}{l}0,0 \\
0,0\end{array}$ & $\begin{array}{l}0,0 \\
0,0\end{array}$ & $\begin{array}{l}0,0 \\
0,0\end{array}$ & $\begin{array}{l}3,9 \\
3,9\end{array}$ & $\begin{array}{l}4,2 \\
4,2\end{array}$ & $\begin{array}{l}4,0 \\
4,0\end{array}$ & $\begin{array}{l}0,0 \\
0,0\end{array}$ & $\begin{array}{l}0,0 \\
0,0\end{array}$ & $\begin{array}{l}0,0 \\
0,0\end{array}$ & $\begin{array}{l}1,3 \\
1,3\end{array}$ \\
\hline Bactris ferruginea Burret & 2,7 & 2,8 & 2,7 & 0,0 & 0,0 & 0,0 & 0,0 & 0,0 & 0,0 & 0,9 \\
\hline Casearia arborea (Rich.) Urb. & 2,7 & 2,8 & 2,7 & 0,0 & 0,0 & 0,0 & 0,0 & 0,0 & 0,0 & 0,9 \\
\hline Luehea ochrophylla Mart. & 2,7 & 2,8 & 2,7 & 0,0 & 0,0 & 0,0 & 0,0 & 0,0 & 0,0 & 0,9 \\
\hline Allophylus edulis Radlk. & 0,0 & 0,0 & 0,0 & 0,0 & 0,0 & 0,0 & 2,3 & 2,4 & 2,4 & 0,8 \\
\hline Cedrela sp. & 0,0 & 0,0 & 0,0 & 0,0 & 0,0 & 0,0 & 2,3 & 2,4 & 2,4 & 0,8 \\
\hline Eschweilera ovata (Cambess.) Miers & 0,0 & 0,0 & 0,0 & 0,0 & 0,0 & 0,0 & 2,3 & 2,4 & 2,4 & 0,8 \\
\hline Guarea guidonia (L.) Sleumer & 0,0 & 0,0 & 0,0 & 0,0 & 0,0 & 0,0 & 2,3 & 2,4 & 2,4 & 0,8 \\
\hline Guatteria pogonopus Mart. & 0,0 & 0,0 & 0,0 & 0,0 & 0,0 & 0,0 & 2,3 & 2,4 & 2,4 & 0,8 \\
\hline Indet. 1 & 0,0 & 0,0 & 0,0 & 0,0 & 0,0 & 0,0 & 2,3 & 2,4 & 2,4 & 0,8 \\
\hline Ouratea cf. hexasperma (A. St.-Hil.) Baill. & 0,0 & 0,0 & 0,0 & 0,0 & 0,0 & 0,0 & 2,3 & 2,4 & 2,4 & 0,8 \\
\hline Sorocea hilarii Gaudich. & 0,0 & 0,0 & 0,0 & 0,0 & 0,0 & 0,0 & 2,3 & 2,4 & 2,4 & 0,8 \\
\hline Total & 100 & 100 & 100 & 100 & 100 & 100 & 100 & 100 & 100 & 100 \\
\hline
\end{tabular}

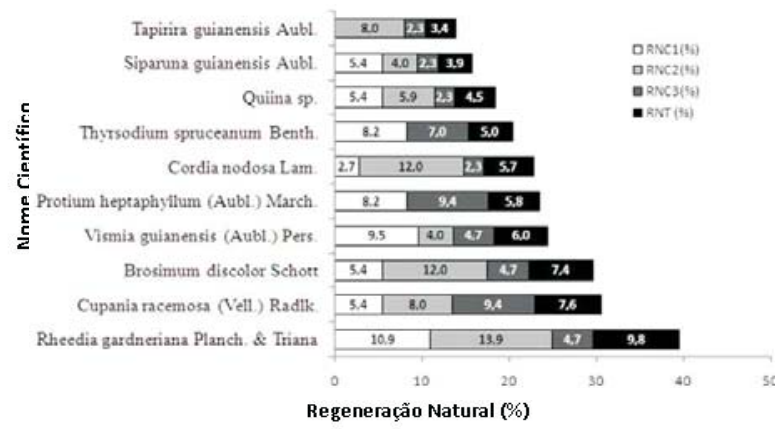

Figura 2 - Relação das 10 espécies que apresentam valores mais altos de regeneração natural total da população amostrada (RNT), expressos em percentagem, amostradas no fragmento Mata Santa Luzia, em Catende, PE.

Figure 2 - Relation of 10 species that show elevated values of total natural regeneration of the samples population (RNT) in percentage, in the Mata Santa Luzia fragment, Catende's city, PE.

Lam. (5,7\%), Thyrsodium spruceanum Benth. (5,1\%), Quiina sp. (4,6\%), Siparuna guianensis Aubl. (4,0\%) e Tapirira guianensis Aubl. (3,5\%), e juntas elas representam cerca de 59,9\% do total (Figura 2), em que a maioria das espécies se comportou como secundárias iniciais, à exceção da Cordia nodosa e Vismia guianensis, que pertencem ao grupo ecológico das espécies pioneiras.
Entre as 36 espécies amostradas, as que apresentaram para Regeneração Natural na Classe 1 (RNC1) maiores percentuais foram Rheedia gardneriana Planch. \& Triana (10,9\%), Vismia guianensis (Aubl.) Pers. (9,6\%) e Protium heptaphyllum (Aubl.) Marchand $(8,2 \%)$ (Figura 3). Para Regeneração Natural na classe 2 (RNC2), a que se destacou foi, Rheedia gardneriana Planch. \& Triana, com 13,9\%, seguida por Brosimum discolor Schott (12\%) e Cordia nodosa Lam. (12\%). Para Regeneração Natural na Classe 3 (RNC3), as espécies que apresentaram maiores valores foram: Protium heptaphyllum (Aubl.) Marchand (9,4\%), Cupania racemosa (Vell.) Radlk (9,4\%) e Thyrsodium spruceanum Benth. (7\%).

\section{DISCUSSÃO}

Os resultados encontrados neste estudo em relação à florística do fragmento quando restritos à família diferem de muitos dos já desenvolvidos na mesma tipologia e com espécies em fase de regeneração natural, por exemplo o trabalho de Nappo et al. (2004), que teve como objetivo avaliar a dinâmica da estrutura fitossociológica da regeneração natural em sub-bosque de Mimosa scabrella Bentham em Minas Gerais. Esses autores observaram que as famílias que apresentaram maior número de espécies arbóreas em fase de 
regeneração foram: Myrtaceae com nove espécies, Melastomataceae com sete, representando 15,38\%, e a Anacardiaceae, Bignoniaceae, Malpighiaceae e Piperaceae (14 espécies no total), representando 22,22\% da regeneração natural.

Souza et al. (2002), trabalhando em fragmento de Floresta Ombrófila Densa secundária no Espírito Santo, visando obter rápido retorno da floresta às suas condições primárias, bem como analisar seus efeitos sobre a dinâmica da composição florística, constataram que, ao longo do período (oito anos), as seguintes famílias que mereceram destaque sob esse aspecto foram: Myrtaceae (45 espécies), Sapotaceae (27), Caesalpiniaceae (25), Fabaceae (17), Mimosaceae (14), Lauraceae (14), Moraceae (12), Euphorbiaceae (11), Flacourtiaceae (11) e Chrysobalanaceae (11).

Estudando a frequência e distribuição das espécies nas classes de regeneração natural em um fragmento de floresta atlântica na região metropolitana do Recife, Silva-Junior (2004) encontrou resultados que corroboram os deste trabalho, em que foi verificada a presença de quatro espécies similares entre as mais destacadas, com maior valor de percentual de regeneração natural total. Esse fato pode ser explicado provavelmente pelo fato de ambos os estudos se situarem em áreas de mesma tipologia florestal e em condições edafoclimáticas similares e de essas espécies serem pioneiras em estágios iniciais de sucessão.

Das 36 espécies, 11 obtiveram valores de RNT inferiores a $1,0 \%$, o que indica, a partir de determinado tempo, que essas espécies podem passar a ter maior grau de dificuldade em se regenerar ou indicar que essas são espécies tardias que estão ingressando no ecossistema.

Analisando os resultados obtidos pela regeneração natural total (RNT) no fragmento estudado, foi observada amplitude variando de 11,2 a 1,13\%, e essa menor amplitude pode ser devida ao fato de esse fragmento estar constantemente sobre pressão antrópica e apresentar, no seu interio,r vestígios diários de depredação.

A presença da maioria das espécies pertencentes ao grupo das pioneiras e secundárias iniciais nas classes de alturas 1 e 2 se deve, provavelmente, às condições favoráveis, de radiação e microclima do fragmento, para o desenvolvimento de espécies mais intolerantes a baixa intensidade de luz, demonstrando que as condições de perturbação influenciam diretamente no início de uma evolução sucessional, o que é um resultado esperado em fragmentos alterados.

O fragmento está situado próximo a comunidades e apresentou um nível de perturbação preocupante, quando comparado com outros de mesma fisionomia na região. O desmatamento pode estar ligado, possivelmente, ao fato de o fragmento estar localizado a $11 \mathrm{~km}$ da via pública, o que auxilia a exploração quase que imperceptível dos recursos da área. Dessa forma, os resultados aqui apresentados permitem presumir que, possivelmente, as espécies encontradas com alta capacidade de regeneração realmente estejam presentes na futura floresta, entretanto ressalta-se que é importante o acompanhamento da área estudada associado ao monitoramento do crescimento das espécies amostradas, de modo a permitir um real entendimento sobre a dinâmica de reposição do dossel.

\section{REFERÊNCIAS}

CONDEPE. Catende. Recife, 1987. (Monografias Municipais, 27). p.62.

CRONQUIST, A. The evolution and classification of flowering plants. $2^{\circ}$. ed., New York: The New York Botanical Garden, 1988, 555p.

EMBRAPA (Empresa Brasileira de pesquisa Agropecuária). Sistema Brasileiro de Classificação dos Solos. 2005.

Disponível em: <http:

www.cnps.embrapa.br/sibcs/>, acesso em: 02. Julho. 2005.

FELFILI, J. M.; REZENDE, R. P. Conceitos e métodos em fitossociologia. Universidade de Brasília, Brasília. 2003. p. 44-53.

FERREIRA, R. L. C. Análise estrutural da vegetação da Estação Florestal da Experimentação de Açú - RN, como subsídio básico para o manejo florestal. 1988. 91p. Dissertação (Mestrado em Ciências Florestais) - Universidade Federal de Viçosa, Viçosa. 
FINOL, U. H. Nuevos parametros a considerarse en el analisis estrutural de las selvas virgenes tropicales. Revista Forestal Venezolana, v.18, n.12, p.29-42. 1971.

GAMA, J. R. V.; BOTELHO, S. A.; BENTESGAMA, M. de M.; Composição florística e estrutura da regeneração natural de floresta secundária de várzea baixa no estuário amazônico. Revista Árvore. Viçosa, v.26, n.5, p.001-011, 2002.

HIGUCHI, N. et al. Bacia 3 - Inventário diagnóstico da regeneração natural. Acta Amazônica. Manaus, v. 15, n. 1/2, p.199-233, 1985.

MARANGON, L. C. Florística e fitossociologia de área de floresta estacional semidecidual visando dinâmica de espécies florestais arbóreas no município de Viçosa, MG. 1999. 139f. Tese (Doutorado em Ecologia e Recursos Naturais) - Universidade Federal de São Carlos, São Carlos, MG.

MARANGON, L. C.; SOARES, J. J.; FELICIANO, A. L. P. Florística Arbórea da Mata da Pedreira, Município de Viçosa, Minas Gerais. Minas Gerais. Revista Árvore. v.27, n.2, 2003. 207-215p.

NAPPO, M. E.; FONTES, M. A. L.; OLIVEIRAFILHO, A. T. de. Suficiência amostral e análise do tamanho de parcelas para o estudo da regeneração natural do sub-bosque de povoamentos homogêneos de Mimosa scabrella Benth., em área minerada, em Poços de Caldas-MG. Revista Árvore, Viçosa, v. 23, n. 4, p. 443-453, 1999.
RONDON NETO, R. M.; BOTELHO, S. A.; FONTESA, M. A. L.; DAVIDE, A. C.; FARIA, J. M. R. Estrutura e Composição Florística da Comunidade Arbustivo-Arbórea de uma Clareira de Origem Antrópica, em uma Floresta Estacional Semidecídua Montana, Lavras-MG, Brasil.

Revista Cerne, Lavras, v.6, n.2, p.79-94. 2000.

SILVA JÚNIOR, J. F. Estudo fitossociológico em um remanescente de floresta atlântica visando dinâmica de espécies florestais arbóreas no município do Cabo de Santo Agostinho, PE. 2004. 84f. Dissertação (Mestrado em Ciências Florestais) Universidade Federal Rural de Pernambuco, Recife.

SILVA, W. C.; MARANGON, L. C.; FERREIRA, R. L. C.; FELICIANO, A. L. P.; COSTA JUNIOR, R. F. Estudo da Regeneração Natural de Espécies Arbóreas em Fragmento Floresta Ombrófila Densa, Mata das Galinhas, no Município de Catende, Zona da Mata Sul de Pernambuco. Revista Ciência Florestal, Santa Maria, v. 17, n. 4, p. 321-331, 2007.

SOUZA A. L. de; SCHETTINO, S; JESUS, R. M. de; VALE, A. B. do. Dinâmica da regeneração natural em uma Floresta Ombrófila Densa secundária, após corte de cipós, reserva natural da Companhia Vale do Rio Doce S.A., estado do Espírito Santo, Brasil. Revista Árvore, Viçosa, v.26, n.4, p.411-419, 2002.

VOLPATO, M. M. L. Regeneração natural em uma floresta secundária no domínio de Mata Atlântica: uma análise fitossociológica. 1994. 123 f. Dissertação (Mestrado em Ciência Florestal) Universidade Federal de Viçosa, Viçosa. 